\title{
Streptozotocin-induced diabetes in rats is associated with impaired metabolic availability of vitamin A (retinol)
}

\author{
BY P. J. TUITOEK ${ }^{1}$, S.ZIARI ${ }^{3}$, A. T. C. TSIN ${ }^{3}$, R. V. RAJOTTE ${ }^{2}$, MIYOUNG SUH ${ }^{1}$ \\ AND TAPAN K. BASU* \\ ${ }^{1}$ Department of Agricultural, Food and Nutritional Science, University of Alberta, Edmonton, \\ Alberta, Canada T6G $2 P 5$ \\ ${ }^{2}$ Surgical-Medical Research Institute, University of Alberta, Edmonton, Alberta, Canada T6G 2N8 \\ ${ }^{3}$ Division of Life Sciences, The University of Texas at San Antonio, San Antonio, Texas,
} 78249-0662, USA

(Received 5 October 1994-Revised 22 May 1995 - Accepted 16 August 1995)

\begin{abstract}
Using streptozotocin-induced diabetic Wistar rats, studies were carried out to examine the metabolic availability of vitamin $A$ in the plasma, liver and the retina of the eye. Control and diabetic rats were fed ad lib. on a semi-purified diet either with or without (basal) vitamin A supplementation, or pair-fed on the basal diet for 4 weeks. Despite the fact that diabetic rats consumed $48 \%$ more feed, they had lower plasma concentrations of retinol $(P<0.003)$. The decrease in plasma retinol concentration was a response to diabetes (or diabetes-induced trauma), since neither pair-feeding $(P<0.01)$ nor vitamin $A$ supplementation altered this effect $(P<\mathbf{0 . 0 5})$. Furthermore, the hepatic concentrations of the vitamin in these animals remained elevated and this increase was greater in the supplemented diabetic group $(P<$ 0.001). Decreases in 11-cis retinal (a component of rhodopsin) concentrations in the retina were also observed in diabetic animals. The increased hepatic and the decreased plasma and retina vitamin $A$ levels suggest a defect in the transport of the vitamin from the liver.
\end{abstract}

Diabetes: Streptozotocin: Vitamin A

Diabetes mellitus is a chronic disease characterized by abnormalities in the metabolism of carbohydrate, lipid, and protein. It is the major cause of retinopathy and is currently a leading cause of blindness in adults (Palmberg, 1977; Moss et al. 1989). Vitamin A plays an essential role in vision; therefore its deficiency may ultimately cause blindness (Oomen, 1974). The mechanisms leading to blindness in vitamin A deficiency and in diabetes are different, but it is conceivable that retinopathy, precipitated by diabetes, can be further intensified by vitamin A deficiency. Elevated serum $\beta$-carotene (a precursor of vitamin A) levels associated with cutaneous signs of carotenaemia have been found to be present in patients with diabetes, suggesting that the diabetic state may interfere with the conversion of carotene to vitamin A (Gouterman \& Sibrack, 1980). Furthermore, $\mathrm{Zn}$ is known to be important in the hepatic synthesis of the vitamin A carrier, retinol-binding protein (RBP) (Smith, 1982), and its metabolism is affected in the presence of diabetes mellitus, as evident by hyperzincuria (Kinlaw et al. 1983; Lau \& Failla, 1984). Recent studies have shown that insulin-dependent diabetic patients have decreased concentrations of both plasma retinol and its carrier protein when compared with non-diabetic subjects (Basu et al. 1989; Krempf et al. 1991; Martinoli et al. 1993). Furthermore, non-insulin-dependent diabetic patients appear to have reduced plasma retinol but elevated levels of retinyl esters, suggesting an increased hepatic storage of vitamin A (Wako et al. 1986).

$$
\text { * For reprints. }
$$


Despite biochemical and clinical associations with diabetes, the metabolism of vitamin A in the disease is not well understood. Therefore using streptozotocin (STZ)-induced diabetic rats, the present study was undertaken to examine the metabolic availability of vitamin $\mathrm{A}$ in the plasma, liver and the retina of the eye.

\section{MATERIALS AND METHODS}

Animals and tissue collections

Male Wistar rats (Crl: (WI)BR) weighing 250-300 $\mathrm{g}$ were obtained from Charles River, Montreal, Canada. They were housed in stainless steel cages in a well-ventilated room maintained at $21^{\circ}$ and were on a $12 \mathrm{~h}$ light-dark cycle. Diabetes was induced by intravenous injection of STZ $(55 \mathrm{mg})$ dissolved in acetate buffer (pH 4.5). Control rats were injected with acetate buffer alone. Following injection with STZ, animals displaying plasma glucose concentrations of greater than $18 \mathrm{mmol} / \mathrm{l}$ were considered diabetic. Plasma glucose concentrations were measured by the glucose oxidase $(E C$ 1.1.3.4) method using the Beckman glucose analyser (Beckman Instruments, Palo Alto, CA, USA) (Kadish et al. 1968). There were three experiments which were carried out for a period of 4 weeks. In Expts 1 and 2, control and diabetic animals were given free access to a semi-purified diet (Table 1) either with or without vitamin A supplementation. In Expt 3, individual rats were pair-fed with the semi-purified diet so that each diabetic rat consumed a daily weight of feed equal to that consumed by control animals of the same weight. Thus each pair of control and diabetic animals received the same amount of vitamin A per day. The vitamin-A supplemented group were given free access to the semi-purified diet but supplemented with ten times more retinyl acetate ( 36 retinol equivalents (RE)/g diet). Experimental protocols were reviewed and approved by the Animal Policy and Welfare Committee, University of Alberta, Canada.

All animals were allowed free access to water and records of feed intake and body weight were kept throughout the study. Animals were killed at the end of 4 weeks, using $\mathrm{CO}_{2}$. Blood was collected in heparinized tubes; separated plasma was protected from light and stored at $-20^{\circ}$, pending analysis. Livers were removed, cleaned and frozen immediately in liquid $\mathrm{N}_{2}$. The retina of the eye was isolated by the method of Fumiyuki et al. (1989). The eyes were enucleated and the anterior segment was removed. After removal of the vitreous body with slight pressure, the retina was detached from the lens, washed in cold saline $(9 \mathrm{~g} \mathrm{NaCl} / \mathrm{l})$ solution and frozen in liquid $\mathrm{N}_{2}$.

Plasma samples were analysed within 1 week and the tissues, including the liver and retina of the eye, within 4 weeks of collection.

\section{Vitamin A analysis}

Plasma and liver vitamin A concentrations were assayed by HPLC using retinyl acetate as the internal standard (Nierenberg \& Lester, 1985). Liver samples were homogenized in nine volumes of water in a Brinkmann Polytron homogenizer (Brinkman, Rexdale, Ontario, Canada) (Leichter et al. 1991). The homogenate was then saponified with an equal volume of $\mathrm{KOH}$ in methanol $(50 \mathrm{~g} / \mathrm{l})$ for $1 \mathrm{~h}$ at $50^{\circ}$. Unsaponified samples of plasma $(200 \mu \mathrm{l})$ and the saponified liver samples $(200 \mu l)$ were extracted with $n$-hexane. The hexane layer was removed, evaporated to dryness with $\mathrm{N}_{2}$ and redissolved into $200 \mu \mathrm{l}$ acetonitrile, which was then injected into the HPLC. A set of retinyl acetate standards were carried through the procedures of saponification (Frolik \& Olson, 1984), resulting in $86 \%$ recovery. Free retinol in the liver was extracted without saponification. Chromatography was performed on a C18 reverse-phase column (Whatman partisil SOD-3, $5 \mu \mathrm{m}$ particle size, $100 \mathrm{~mm}$; Whatman Inc., Clifton, NJ, USA), by use of a mobile phase consisting of methanol-water 
Table 1. Composition of the semi-purified diet*

\begin{tabular}{llc}
\hline \multicolumn{1}{c}{ Ingredient $\dagger$} & $(\mathrm{g} / \mathrm{kg})$ \\
\hline Casein (vitamin free) & 200 \\
Maize starch & 600 \\
Non-nutritive bulk & 50 \\
Maize oil & 100 \\
AIN vitamin mix (with choline) $\ddagger$ & $12 \cdot 5$ \\
AIN mineral mixture $\ddagger$ & 35 \\
DL-Methionine & $2 \cdot 5$ \\
\hline
\end{tabular}

* $A d$ lib. and pair-fed groups received retinol acetate, $4.13 \mu \mathrm{g} / \mathrm{g}$ (3.6 retinol equivalents). The vitamin Asupplemented diet contained 36 retinol equivalents $/ g$.

$\dagger$ Ingredients were from ICN Biochemicals, Cleveland, Ohio, USA.

$\ddagger$ American Institute of Nutrition $(1977,1980)$.

Table 2. Vitamin A status of non-supplemented control and diabetic rats* (Mean values with their standard errors for six rats per group)

\begin{tabular}{|c|c|c|c|c|c|}
\hline & \multicolumn{2}{|c|}{ Control } & \multicolumn{2}{|c|}{ Diabetic } & \multirow[b]{2}{*}{$P$ value } \\
\hline & Mean & $\mathrm{SE}$ & Mean & SE & \\
\hline Total body-weight (BW) gain (g) & 185 & 13 & 24 & 16 & 0.0001 \\
\hline Liver weights $(\mathrm{g} / \mathrm{kg} \mathrm{BW})$ & $32 \cdot 8$ & 0.7 & $44 \cdot 4$ & 0.7 & 0.0001 \\
\hline Plasma glucose $(\mathrm{mmol} / \mathrm{l})$ & 6.00 & 0.24 & $21 \cdot 6$ & 1.91 & 0.0001 \\
\hline Feed intake $(\mathrm{g} / \mathrm{d})$ & $23 \cdot 4$ & 0.94 & $34 \cdot 0$ & 0.95 & 0.0001 \\
\hline Vitamin A intake (RE/d) & $84 \cdot 2$ & 3.40 & 122 & $3 \cdot 40$ & 0.0001 \\
\hline Plasma retinol $(\mu \mathrm{mol} / 1)$ & 1.35 & $0 \cdot 10$ & 0.91 & 0.05 & 0.003 \\
\hline Hepatic vitamin A $(\mu \mathrm{mol} / \mathrm{g})$ & $0 \cdot 50$ & 0.01 & 0.99 & 0.05 & 0.0001 \\
\hline
\end{tabular}

* Diets contained 3.6 retinol equivalents (RE)/g. (1 RE $=1 \mu \mathrm{g}$ retinol.)

$(95: 5, \mathrm{v} / \mathrm{v})$. Detection was by u.v. absorption at $325 \mathrm{~nm}$, and quantification was performed by comparison of peak areas produced by the injection of known amounts of retinol.

Retina samples were analysed for 11-cis retinal using a modification of the method outlined by Suzuki et al. (1986). A whole retina was homogenized in $200 \mu$ l sodium phosphate buffer $(0.1 \mathrm{M}, \mathrm{pH} 6.8)$ with a glass-teflon minihomogenizer. The homogenate was mixed with $100 \mu \mathrm{l}$ formaldehyde $(370 \mathrm{ml} / \mathrm{l})$, left at room temperature for $5 \mathrm{~min}$ and then mixed with $200 \mu \mathrm{l}$ isopropanol. Distilled water $(0.5 \mathrm{ml})$ and $3 \mathrm{ml} n$-hexane were added to the sample mixture and it was shaken vigorously. After centrifugation at $3000 \mathrm{rev} . / \mathrm{min}$ for $5 \mathrm{~min}$, the hexane upper phase was collected. The extraction with $n$-hexane was repeated three times. The extracts were combined, evaporated to dryness with $\mathrm{N}_{2}$ at $50^{\circ}$, redissolved into $200 \mu \mathrm{l}$ of mobile phase ( $n$-hexane containing $30 \mathrm{ml}$ dioxane $/ 1$ ), which was injected into the HPLC. Chromatography was performed in a Zorbax SIL column ( $7 \mu \mathrm{m}$ particle size, $2 \cdot 1 \times 250 \mathrm{~mm}$; Dupont de Nemours and Co., Wilmington, DE, USA).

\section{Statistical analysis}

Data were tabulated to give means with their standard errors using the SAS computer program (Statistical Analysis Systems Institute, 1988). To determine differences between 


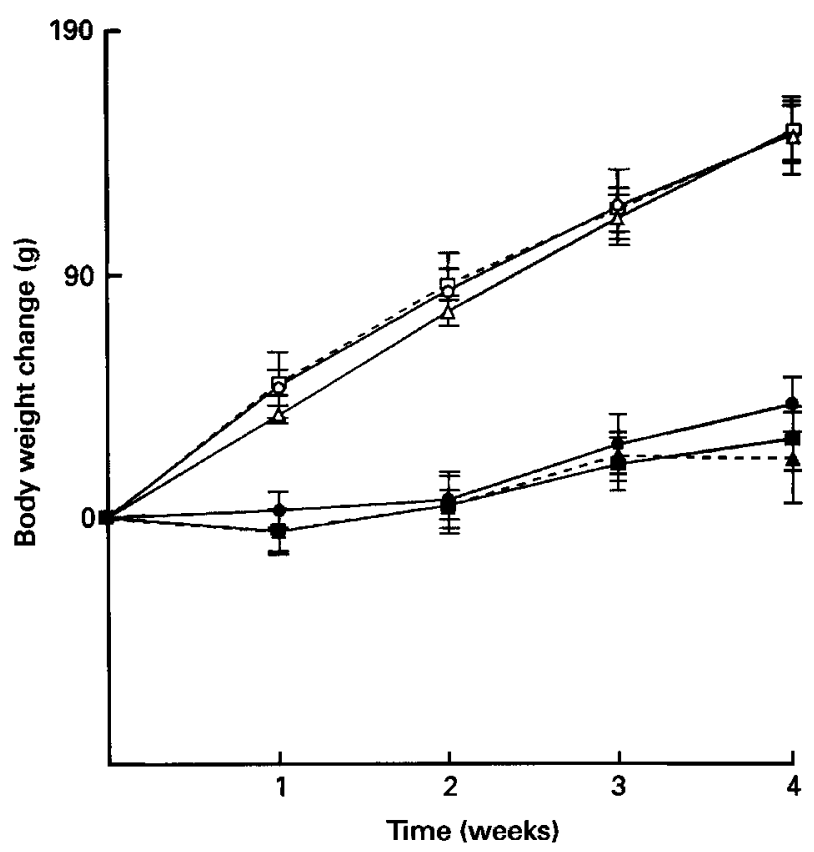

Fig. 1. Weekly body-weight changes in streptozotocin-induced diabetic rats fed ad lib. on diets with (control $\mathrm{O}$, diabetic - ) or without (control $\square$, diabetic $\square$ ) vitamin A supplementation, or pair-fed without vitamin A supplementation (control $\Delta$, diabetic $\Delta$ ). Values are means for six rats, with their standard errors indicated by vertical bars.

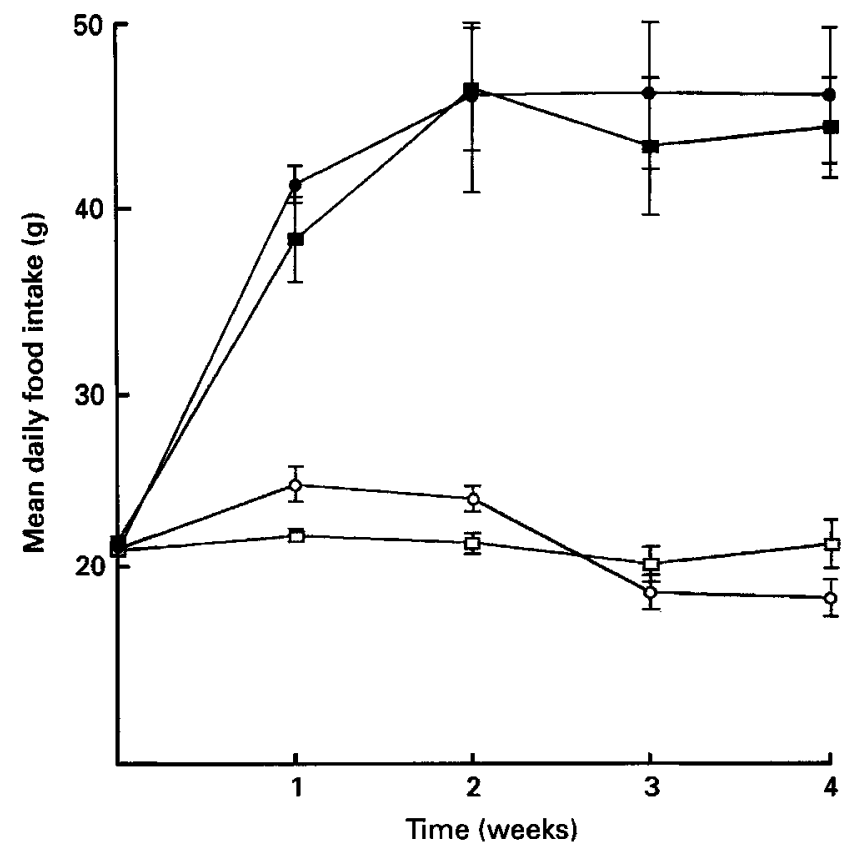

Fig. 2. Daily food intakes of $a d$ lib.-fed streptozotocin-induced diabetic rats with (control $\square$, diabetic $\mathbf{\square}$ ) and without (control $O$, diabetic $\bigcirc$ ) vitamin A supplementation. Values are means for six rats, with their standard errors indicated by vertical bars. 
Table 3. Effect of vitamin $A$ supplementation for 4 weeks on the plasma and hepatic concentrations of vitamin $A$ in control and diabetic rats*

(Mean values with their standard errors for six rats per group)

\begin{tabular}{|c|c|c|c|c|c|}
\hline & \multicolumn{2}{|c|}{ Control } & \multicolumn{2}{|c|}{ Diabetic } & \multirow[b]{2}{*}{$P$ value } \\
\hline & Mean & SE & Mean & SE & \\
\hline Total body-weight (BW) gain (g) & 185 & 16 & 53 & 13 & 0.0001 \\
\hline Liver weights $(\mathrm{g} / \mathrm{kg} \mathrm{BW})$ & $34 \cdot 7$ & 1.7 & $42 \cdot 4$ & $1 \cdot 1$ & 0.003 \\
\hline Plasma glucose $(\mathrm{mmol} / \mathrm{l})$ & 6.20 & 0.14 & $22 \cdot 7$ & $3 \cdot 15$ & 0.0004 \\
\hline Feed intake $(\mathrm{g} / \mathrm{d})$ & 21.9 & 0.73 & $34 \cdot 7$ & $2 \cdot 19$ & 0.0002 \\
\hline Vitamin A intake (RE/d) & 871 & 29.7 & 1377 & $86 \cdot 1$ & 0.0002 \\
\hline Plasma retinol $(\mu \mathrm{mol} / 1)$ & $1 \cdot 18$ & 0.07 & 0.96 & 0.08 & 0.05 \\
\hline Hepatic vitamin A $(\mu \mathrm{mol} / \mathrm{g})$ & 2.64 & 0.18 & $5 \cdot 21$ & 0.42 & 0.0001 \\
\hline
\end{tabular}

* Diets were supplemented with vitamin A (36 retinol equivalents (RE)/g). For further details of diets and procedures, see Table 1 and pp. 616-617.

group means, Student's $t$ test was used for the non-supplemented and the vitamin Asupplemented groups, and a paired $t$ test was used for the pair-fed group (Steel \& Torrie, 1980). The upper level of significance was preset at $P<0.05$.

\section{RESULTS}

The STZ-induced diabetic rats given free access (ad lib. group) to the semi-purified diet without (Table 2) or with supplementation (Table 3) of vitamin A for 4 weeks exhibited elevated plasma glucose concentrations with no difference in the two experimental groups. This hyperglycaemia was accompanied by glycosuria which became manifest within $48 \mathrm{~h}$, and persisted throughout the 4 week follow-up period. The diabetic animals gained weight but at a slower rate than did the corresponding controls (Fig. 1), even though they consumed significantly more feed (Fig. 2 ).

Despite polyphagia that resulted in a $48 \%$ greater intake of vitamin A, plasma retinol concentrations were significantly lower in the diabetic animals than in the controls $(P<$ 0.003 ) (Table 2). However, the hepatic concentrations of vitamin A were markedly greater in the diabetic animals $(P<0.0001)$. To try and correct for this decreased plasma retinol concentration, in Expt 2 the diabetic rats were fed on a diet supplemented with vitamin A (36 RE/g) which was ten times that present in the basal diet. Even after 4 weeks of this supplementation the circulatory level of vitamin A was lower $(P<0.05)$, while hepatic concentrations increased at this supplemental level compared with the control animals $(P<0.0001)$ (Table 3$)$.

In the pair-fed group, diabetic and control rats were fed isoenergetically on a semipurified diet (Table 1) with the same amount of vitamin A for 4 weeks. These pair-fed animals had similar hepatic concentrations of the vitamin and yet plasma concentrations remained significantly lower in the diabetic rats $(P<0.01)$ (Table 4$)$. The free retinol concentration in the liver however was significantly higher in the diabetic than in the control animals $(P<0.001)$. In the retina the concentrations of 11-cis retinal, an important constituent of rhodopsin (a visual pigment), were found to be significantly lower in the diabetic animals than in the controls $(P<0.05)$. 
Table 4. Vitamin A status of pair-fed control and diabetic rats*

(Mean values and standard errors for six rats per group)

\begin{tabular}{|c|c|c|c|c|c|}
\hline & \multicolumn{2}{|c|}{ Control } & \multicolumn{2}{|c|}{ Diabetic } & \multirow[b]{2}{*}{$P$ value } \\
\hline & Mean & SE & Mean & SE & \\
\hline Body-weight (BW) gain (g) & 159 & 12 & 25 & 19 & 0.0001 \\
\hline Liver weights (g/ $\mathrm{kg} \mathrm{BW})$ & $33 \cdot 5$ & 0.6 & $44 \cdot 1$ & $2 \cdot 3$ & 0.002 \\
\hline Feed intake $(\mathrm{g} / \mathrm{d})$ & $21 \cdot 0$ & 0.95 & $21 \cdot 0$ & 0.85 & $0 \cdot 88$ \\
\hline Vitamin A intake (RE/d) & $75 \cdot 7$ & $2 \cdot 91$ & $75 \cdot 6$ & $2 \cdot 94$ & 0.98 \\
\hline Plasma retinol $(\mu \mathrm{mol} / 1)$ & $1 \cdot 16$ & 0.06 & 0.65 & 0.08 & 0.01 \\
\hline Hepatic vitamin A $(\mu \mathrm{mol} / \mathrm{g})$ & $0 \cdot 48$ & 0.02 & 0.51 & 0.03 & $0 \cdot 11$ \\
\hline Free retinol $(\mu \mathrm{mol} / \mathrm{g}$ liver $)$ & 0.018 & 0.002 & 0.067 & 0.008 & 0.001 \\
\hline 11-cis retinal (pmol/retina) & $52 \cdot 8$ & $16 \cdot 5$ & $22 \cdot 1$ & $3 \cdot 3$ & 0.05 \\
\hline
\end{tabular}

* Diets contained 3.6 retinol equivalents (RE) retinyl acetate $/ g$. For further details of diets and procedures, see Table 1 and pp. 616-617.

\section{DISCUSSION}

The results presented here clearly demonstrate that rats made diabetic with STZ have significantly reduced concentrations of plasma retinol. The results are in agreement with earlier human studies in which plasma retinol concentrations were shown to be significantly lower in diabetic patients than in non-diabetic controls (Basu et al. 1989; Krempf et al. 1991; Martinoli et al. 1993). Despite the decrease in plasma retinol concentrations, liver concentrations of total vitamin A were significantly elevated in the diabetic animals. These animals consumed $48 \%$ more feed than the corresponding controls. Since the hepatic stores of vitamin A are thought to be proportional to daily intake (Hicks et al. 1984), it seems possible that the increased intake of vitamin $\mathbf{A}$ by the diabetic animals accounts, at least in part, for their elevated hepatic concentrations. However, the fact remains that the diabetes-associated reductions in plasma vitamin A levels were not due to the depletion of hepatic stores. In order to determine if decreased plasma retinol concentrations in diabetic animals could be increased to normal values, diabetic animals were fed on a diet which contained ten times the basal diet level of vitamin A (36 RE/g) for 4 weeks. At this augmented intake the plasma concentrations of vitamin $A$ were lower in the diabetic animals, while the hepatic levels increased compared with the control group.

In the pair-feeding experiment, in which the control and diabetic rats were given equal amounts of vitamin A, the plasma retinol remained low in the diabetic animals. However, unlike the diabetic animals given free access to feed, the total hepatic concentration of vitamin $\mathbf{A}$ in the pair-fed diabetic rats was similar to that in the control animals. Since there was no difference in the hepatic concentration of total vitamin A between the pair-fed animals, it is plausible that absorption of vitamin $\mathbf{A}$ is not affected by diabetes. This has been further supported by a recent in vitro study where no difference in the rate of either jejunum or ileum uptake of retinol was observed between the STZ-induced diabetic $v$. control animals (Tuitoek et al. 1994).

The results from the present study suggest that there may be an impairment in the availability of vitamin A from hepatic stores in the presence of diabetes. Vitamin A is stored in the liver as retinyl ester (Blomhoff et al. 1991). It is hydrolysed by retinyl ester hydrolase (EC 3.1.1.64), and carried as free retinol to the circulation and subsequently to the target tissues by RBP (Goodman, 1974). It is important to note that although the pair-fed diabetic 
and control animals showed no difference in their hepatic concentrations of vitamin A (i.e. free retinol + retinyl ester), the diabetic animals exhibited an increased level of free retinol. It is, therefore, unlikely that the availability of vitamin $\mathrm{A}$ in diabetes is affected because of a decreased hepatic hydrolysis of the retinyl esters. An increase in hepatic free retinol levels accompanied by depressed circulatory levels in the diabetic animals suggests that there may be an impaired mobilization of the vitamin from its hepatic stores into the circulation, possibly due to inadequate synthesis of RBP in the liver. Studies in human diabetic subjects indeed show that there is a parallel decrease in plasma RBP with a decrease in plasma retinol (Basu et al. 1989; Krempf et al. 1991; Martinoli et al. 1993).

Lowered levels of plasma vitamin A have been linked with deficiency of $\mathrm{Zn}$ which is required for the synthesis of RBP (Smith, 1982). Hyperzincuria is a common feature of diabetes (Kinlaw et al. 1983; Lau \& Failla, 1984), which may lead to Zn deficiency. It has been shown that in situations where abnormal dark adaptation is accompanied by poor $\mathrm{Zn}$ status, such as in the alcoholic cirrhosis, vitamin A supplementation alone does not improve the poor dark adaptation (Morrison et al. 1978); however in this experiment normalization was accomplished when vitamin A was supplemented with $\mathrm{Zn}$. It has further been shown that STZ-induced diabetes is associated with depressed protein synthesis in the liver (McNurlan \& Garlick, 1981). Although this effect refers to non-specific proteins, there have been studies reporting depressed serum concentrations of RBP and transthyretin (TTR) without affecting albumin and transferrin status, in subjects with insulin-dependent diabetes mellitus (Basu et al. 1989; Kemp \& Frindik, 1991). It is, therefore, possible that synthesis of RBP may be affected by secondary abnormalities in the metabolism of protein and $\mathrm{Zn}$ in the diabetic state.

Vitamin $A$ is important in the visual cycle where 11-cis retinal is utilized by the photoreceptors (Bok, 1990). This involves a series of events that begin with photobleaching of rhodopsin to form opsin and all-trans-retinal, the production of various retinol derivatives, the regeneration of 11-cis retinal and ultimately, the regeneration of the photopigment itself. In the present study, also, the retinas of the eyes of the diabetic rats contained reduced contents of 11-cis retinal when compared with those of the non-diabetic controls. This depressed retinal status may be a consequence of the decreased metabolic availability of vitamin $A$ as indicated by its elevated hepatic and depressed circulatory levels.

We conclude from the present study that STZ-induced diabetes is associated with depressed vitamin A status as shown by the decreased levels of plasma retinol and 11-cis retinal in the retina. The mechanism for this abnormality is not yet fully understood. There may be inadequate synthesis of RBP in the liver, decreased stability (shorter half-life), or increased degradation of either mRNA or protein for RBP. Future work needs to look at the effect of diabetes on the transport of the vitamin. It is important to note that the decreased levels of plasma retinol were not improved by vitamin A supplementation. The depressed levels of plasma retinol and subsequently its unavailability to the retina may further aggravate the complication found in the retina that is often associated with the diabetic state. Since vitamin A supplementation resulted in a further increase of the vitamin in the liver it may also lead to hepatotoxicity due to an excessive load of the vitamin. A recent case report (Kowalski et al. 1994) describing fatal hepatotoxicity in a 45-year-old diabetic subject consuming $7.5 \mathrm{mg}$ vitamin A daily for more than 6 years may substantiate this possible clinical consequence. The effects observed in the present study were seen only after 4 weeks of diabetes. In the clinical situation, diabetes is a chronic condition, hence the results of the study are of importance. It should be pointed out, however, that although vitamin A supplementation may be potentially hepatotoxic to diabetics, it is very unlikely that the toxic effect would result from intake of the vitamin from normal dietary sources. 


\section{REFERENCES}

American Institute of Nutrition (1977). Report of the American Institute of Nutrition ad hoc committee on standards for nutritional studies. Journal of Nutrition 107, 1340-1348.

American Institute of Nutrition (1980). Second report of the ad hoc committee on standards for nutritional studies. Journal of Nutrition 110, 1726.

Basu, T. K., Tze, W. J. \& Leichter, J. (1989). Serum vitamin A and retinol-binding protein in patients with insulindependent diabetes mellitus. American Journal of Clinical Nutrition 50, 329-331.

Blomhoff, R., Green, M. H., Green, J. B., Berg, T. \& Norum, K. R. (1991). Vitamin A metabolism: new perspectives on absorption, transport, and storage. Physiological Reviews 71, 951-990.

Bok, D. (1990). Processing and transport of retinoids by the retinal pigment epithelium. Eye 4, 326-332.

Frolik, C. H. \& Olson, J. A. (1984). Extraction, separation and chemical analysis of retinoids. In The Retinoids, vol. 1, pp. 181-233 [M. B. Sporn, A. B. Roberts and D. S. Goodman, editors]. Orlando, FL: Academic Press.

Fumiyuki, V., Yasumura, D. \& La Vail, M. M. (1989). New isolations of retina and interphoto receptor matrix. Experimental Eye Research 49, 305-309.

Goodman, D. S. (1974). Vitamin A transport and retinol-binding protein metabolism. Vitamins and Hormones 32 , $167-180$

Gouterman, I. H. \& Sibrack, L. A. (1980), Cutenous manifestation of diabetes. Cutis 25, 45-54.

Hicks, V. A., Gunning, D. B. \& Olson, J. A. (1984). Metabolism, plasma transport and biliary excretion of radioactive vitamin $\mathbf{A}$ and its metabolites as a function of liver reserves of vitamin $\mathrm{A}$ in the rat. Journal of Nutrition 114, 1327-1333.

Kadish, A. H., Little, R. L. \& Sternberg, J. C. (1968). A new and rapid method for the determination of glucose by measurement of rate of oxygen consumption. Clinical Chemistry 14, 116-131.

Kemp, S. F. \& Frindik, J. P. (1991). Effect of metabolic control on serum protein concentrations in diabetes. Acta Paediatrica Scandinavica 80, 938-943.

Kinlaw, W. B., Levine, A. S., Morley, J. E., Silvis, S. E. \& McClain, C. J. (1983). Abnormal zinc metabolism in type II diabetes mellitus. American Journal of Medicine 75, 273-277.

Kowalski, T. E., Falestiny, M., Furth, E. \& Malet, P. F. (1994). Vitamin A hepatoxicity: a cautionary note regarding 25,000 IU supplements. American Journal of Medicine 97, 523-528.

Krempf, M., Ranganathan, S., Ritz, P., Morin, M. \& Charbonnel, B. (1991). Plasma vitamin A and E in type 1 and type 2 adult diabetic patients. International Journal for Vitamin and Nutrition Research 61, 38-42.

Lau, A. \& Failla, M. L. (1984). Urinary excretion of zinc, copper and iron in the streptozotocin-induced diabetic rat. Journal of Nutrition 114, 224-233.

Leichter, J., McNeill, J. H. \& Basu, T. K. (1991). Influence of insulin on plasma and liver vitamin A levels in diabetic rats. Journal of Clinical Biochemistry and Nutrition 11, 47-52.

McNurlan, M. A. \& Garlick, P. J. (1981). Protein synthesis in liver and small intestine in protein deprivation and diabetes. American Journal of Physiology 241, E238-E245.

Martinoli, L., DiFelice, M., Seghieri, G., Ciuti, M., De Giorgio, L. A., Fazzini, A., Gori, R., Anachini, R. \& Franconi, F. (1993). Plasma retinol and $\alpha$-tocopherol concentration in insulin-dependent diabetes mellitus: their relationship to microvascular complications. International Journal for Vitamin and Nutrition Research $\mathbf{6 3}$, $87-92$.

Morrison, S. A., Russel, R. M., Carney, E. A. \& Oaks, E. V. (1978). Zinc deficiency: a cause of abnormal dark adaptation in cirrhotics. American Journal of Clinical Nutrition 31, 278-281.

Moss, S. E., Klein, R. \& Klein, B. E. K. (1989). The incidence of vision loss in a diabetic population. Opthalmology 95, 1340-1348.

Nierenberg, D. W. \& Lester, D. C. (1985). Determination of vitamin A and E in serum and plasma using a simplified clarification method and high-performance liquid chromatography. Journal of Chromatography 345 , 275-284.

Oomen, H. A. P. C. (1974). Vitamin A deficiency, xerophthalmea and blindness. Nutrition Reviews 32, 161-170. Palmberg, P. F. (1977). Diabetic retinopathy. Diabetes 26, 703-709.

Smith, J. C. (1982). Interrelationship of zinc and vitamin A metabolism in animal and human nutrition: a review. In Clinical, Biochemical and Nutritional Aspects of Trace Elements, pp. 239-258. New York: Alan R. Liss Inc.

Statistical Analysis Systems (1988). SAS User's Guide, Statistics. Cary, NC: SAS Institute Inc.

Steel, R. G. D. \& Totrie, J. H. (1980). Principles and Procedures of Statistics: A Biometrical Approach, pp. 67-119. New York: McGraw-Hill.

Suzuki, T., Fujita, Y., Noda, Y. \& Miyata, S. (1986). A simple procedure for the extraction of the mature chromophore of visual pigments: the formaldehyde method. Vision Research 26, 425-429.

Tuitoek, P. J., Thomson, A. B. R., Rajotte, R. V. \& Basu, T. K. (1994). Intestinal absorption of vitamin A in streptozotocin-induced diabetic rats. Diabetes Research 25, 151-158.

Wako, Y., Suzuki, K., Goto, Y. \& Kimura, S. (1986). Vitamin A transport in plasma of diabetic patients. Tohoku Journal of Experimental Medicine 149, 133-143. 